\title{
The spectrum of the cosmic X-ray background observed by RTXE/PCA
}

\author{
M. Revnivtsev ${ }^{1,2}$, M. Gilfanov ${ }^{1,2}$, R. Sunyaev ${ }^{1,2}$, K. Jahoda ${ }^{3}$, and C. Markwardt ${ }^{3}$ \\ 1 Max-Planck-Institute für Astrophysik, Karl-Schwarzschild-Str. 1, 85740 Garching bei München, Germany \\ 2 Space Research Institute, Russian Academy of Sciences, Profsoyuznaya 84/32, 117810 Moscow, Russia \\ 3 Laboratory for High Energy Astrophysics, Code 662, Goddard Space Flight Center, Greenbelt, MD 20771, USA
}

Received 24 June 2003 / Accepted 29 August 2003

\begin{abstract}
We have analyzed a large set of RXTE/PCA scanning and slewing observations performed between April 1996 and March 1999. We obtained the 3-20 keV spectrum of the cosmic X-ray background (CXB) by subtracting Earth-occulted observations from observations of the X-ray sky at high galactic latitude and far away from sources. The sky coverage is approximately $\sim 22.6 \times 10^{3} \mathrm{deg}^{2}$. The PCA spectrum of CXB in 3-20 keV energy band is adequately approximated by a single power law with photon index $\Gamma \sim 1.4$ and normalization at $1 \mathrm{keV} \sim 9.5 \mathrm{phot} / \mathrm{s} / \mathrm{cm}^{2} / \mathrm{keV} / \mathrm{sr}$. Instrumental background uncertainty precludes accurate RXTE/PCA measurements of the spectrum of cosmic X-ray background at energies above $15 \mathrm{keV}$ and therefore we cannot detect the high energy cutoff observed by the HEAO-1 A2 experiment. Deep observations of the 6 high latitude points used to model the PCA background provide a coarse measure of the spatial variation of the CXB. The CXB variations are consistent with a fixed spectral shape and variable normalization characterized by a fractional rms amplitude of $\sim 7 \%$ on angular scales of $\sim 1$ square deg.
\end{abstract}

Key words. cosmology: observations - diffuse radiation - X-rays: general

\section{Introduction}

Many efforts over the last few decades have contributed to an understanding of the origin of the cosmic X-ray background (CXB) in the 2-10 keV band (e.g. Boldt 1987; Hasinger et al. 1991; Fabian \& Barcons 1992; Mushotzky et al. 2000; Giacconi et al. 2002; Brandt et al. 2003). Most, if not all, of the CXB emission is explained by the superposition of point sources (AGNs) distributed over the Universe (see e.g. Rees 1980; Giacconi \& Zamorani 1987; Setti \& Woltjer 1989; Giacconi et al. 2002; Moretti et al. 2003).

Accurate measurements of the X-ray spectrum of the CXB were obtained from large-solid-angle measurements with collimated spectrometers aboard the HEAO- 1 observatory. The HEAO-1 A2 experiment was designed specifically for this problem, with special care being taken to separate the signal from cosmic and instrumental backgrounds. The A2 proportional counter measurements from $\sim 3-60 \mathrm{keV}$ (Marshall et al. 1980) are extended to $1 \mathrm{MeV}$ with the scintillators of the A4 experiment (Gruber 1992). Numerous other measurements have been made with imaging telescopes - EINSTEIN, ROSAT, ASCA, BeppoSAX, CHANDRA, and XMM (e.g. $\mathrm{Wu}$ et al. 1991; Gendreau et al. 1995; Chen et al. 1996; Miyaji et al. 1998; Vecchi et al. 1999; Mushotzky et al. 2000;

Send offprint requests to: $\mathrm{M}$. Revnivtsev, e-mail: mikej@mpa-garching.mpg.de
Lumb et al. 2002). The HEAO-1 A2 measurements were made over a large solid angle with an instrument designed to ensure a precise instrumental background subtraction. The imaging experiments measured the CXB over a much smaller solid angle and could be subject to cosmic variance (see e.g. Barcons 1992; Barcons et al. 2000). The measurements made by X-ray telescopes all yield an absolute normalization significantly larger than that of HEAO-1 A2, a result difficult to explain by cosmic variance alone (e.g. Barcons et al. 2000)

The Proportional Counter Array (PCA) aboard Rossi X-ray Timing Explorer (RXTE) provides an opportunity to perform a new and independent measurement of the CXB spectrum based on nearly all sky data - the first such measurement since the HEAO-1 observations.

\section{Data analysis and results}

\subsection{Data and software}

The Rossi X-ray Timing Explorer (Bradt et al. 1993) carries three instruments including the X-ray spectrometer: the Proportional Counter Array (PCA). It consists of 5 independent Proportional Counter Units (PCUs) which are sensitive to photons in 2-60 keV energy range. For Crab-like spectra, $88 \%$ of the detected counts are below $10 \mathrm{keV}$. Due to its high effective area $\left(\sim 6400 \mathrm{~cm}^{2}\right.$ at $\left.6-7 \mathrm{keV}\right)$, relatively precise 
modeling of the instrumental background, and low deadtime, the PCA can reliably measure spectra for sources with flux greater than $1 \mathrm{mCrab}$, which is about the flux of the CXB integrated over the 1 degree beam of the PCA.

The RXTE is capable of fast slews (6 deg/min); typical operations include 1-2 slews per orbit. Although operations are planned to slew during the South Atlantic Anomaly or periods when targets are occulted to the greatest extent possible, a substantial amount of blank sky and dark earth occulted data is obtained. In addition, several Guest observer programs requested scanning observations over moderate areas of the sky. The slewing and scanning data can be used to construct maps of the sky with the $\sim 1$ deg resolution of the PCA collimator. (e.g. Revnivtsev \& Sunyaev 2002). Scanning RXTE/PCA observations are very useful for the localization of newly discovered sources (Markwardt et al. 2000) and for the study of extended structures on the X-ray sky, especially at relatively high energies (10-20 keV), where only a limited amount of data exists (see e.g. Valinia \& Marshall 1998; Revnivtsev 2003).

In our study we used RXTE/PCA data taken during reorientations (slews or scans) from April 16, 1996 through March 22, 1999. This time period was chosen to stay within a single high voltage epoch of PCA. The total number of observations is approximately 17600 with $\sim 8.5 \mathrm{Msec}$ of exposure. These data contain both clean-sky observations and Earth-occulted observations. Clean-sky data provide the cosmic X-ray background signal, while Earth-occulted data provide information about the PCA instrument background.

The first attempts to model the PCA background used earth-looking data as an estimate of the instrument background. No separation between dark and sunlit earth was made. The estimated CXB spectrum, using this background estimator, deviated from a power law with index $\sim-1.4$ at $\sim 15 \mathrm{keV}$, and also some soft component appeared, an effects that were attributed to reflection from the bright earth. Experience with BBXRT and ASCA (albeit at lower energies) suggests that the sunlit earth is more than an order of magnitude brighter than the dark earth. As we have taken care to include only darkearth data, and as the statistically significant signal extends only to $15-20 \mathrm{keV}$, we are confident that the earth albedo is effectively zero for this experiment. At these low energies the Earth atmosphere, consisting of nitrogen and oxygen, is a very effective absorber, however at energies higher than $10-15 \mathrm{keV}$ the effect of reflection from the atmosphere plays an important role (see e.g. Pendleton et al. 1992) and should be taken into account.

Our analysis assumes that the dark Earth emits essentially zero flux in X-rays. In reality the emission of the dark Earth at higher photon energies is modified by the reflection of cosmic $\mathrm{X}$-ray background and radiation from brightest X-ray sources. However below we would assume that the influence of this effect is small in the spectral band of our interest.

Data reduction was done using standard tools of LHEASOFT 5.2 package. We analyzed data from PCA detectors PCU 0, 1 and 2, which have the largest exposure times. The Noisy parts of the data were filtered out by applying the selection criteria ELECTRON_0, $1,2<0.1$. All results were corrected for the deadtime (http://legacy.gsfc.nasa. gov/docs/xte/recipes/pca_deadtime.html).

The effective field of view of the PCA is $\Omega=2.97 \times 10^{-4} \mathrm{sr}$. This value is derived by fitting scans over the Crab nebula to a model which convolves the response of a perfect hexagonal collimator ( 8 inches high with a $1 / 8$ inch flat to flat opening) with a Gaussian (FWHM 6 arcmin, Jahoda et al. 1996). This model is appropriate for many independent and nearly co-aligned hexagonal collimators; the width of the Gaussian characterizes the average misalignment. While construction of the PCA collimators from corrugated sheets soldered together causes strong correlations between nearby collimator cells, the model works adequately to describe the ensemble of 20000 collimator cells per PCU.

To check the overall normalization (i.e. net area) of the PCA we analyzed the Crab monitoring observations obtained approximately every two weeks over the entire period during which CXB data was collected. We selected data from the same anodes as described above (layer 1, PCUs 0,1 and 2). With the standard response matrix, produced by the FTOOLS 5.2 package the photon index of the Crab spectrum in the $3-20 \mathrm{keV}$ energy band was measured to be $\Gamma=2.09 \pm 0.01$ with a normalization of $N=11.6 \pm 0.4 \mathrm{phot} / \mathrm{s} / \mathrm{cm}^{2} / \mathrm{keV}$ (the value of the neutral absorption column was fixed at $N_{\mathrm{H}}=2 \times 10^{21} \mathrm{~cm}^{-2}$; the results in the PCA band are insensitive to this).

This fit predicts a Crab nebula flux of $2.66 \times 10^{-8} \mathrm{erg} / \mathrm{s} / \mathrm{cm}^{2}$ $(2-10 \mathrm{keV})$ which is high compared to the "conventional" value. Zombeck (1990) reports the Crab spectrum as presented by Seward (1978) to be $\mathrm{d} N / \mathrm{d} E=10 E^{-2.05} \mathrm{phot} / \mathrm{s} / \mathrm{cm}^{2} / \mathrm{keV}$, which gives a $2-10 \mathrm{keV}$ flux of $2.39 \times 10^{-8} \mathrm{erg} / \mathrm{s} / \mathrm{cm}^{2}$. To our knowledge, more recent $\mathrm{X}$-ray experiments have not measured this normalization directly, using instead this value as a standard candle. To put our measurements on this scale, we correct our measured fluxes downward by a factor of 1.11 ; this is equivalent to increasing the estimated geometric area of the PCA ${ }^{1}$. A multi-mission attempt to use type I X-ray bursts as standard candles reached a similar conclusion (Kuulkers et al. 2003) using an earlier version of the PCA response matrix.

Deviations between the modeled and measured Crab spectrum do not exceed $\sim 1 \%$. In all subsequent analysis we cite only statistical uncertainties unless otherwise noted. The quoted uncertainties are consistent with the statistical distributions of measured quantities.

We used the faint source ("L7_240") CM background model (http://heasarc.gsfc.nasa.gov/docs/ xte/recipes/pcabackest.html). The background model includes by design both the cosmic and instrumental background, so the background subtracted rate for "blank sky" observations should be approximately zero. The background model is constructed from observations of six different blank

\footnotetext{
1 We (the GSFC co-authors) expect that the FTOOLS 5.3 package, anticipated for fall 2003 release, will increase the geometric area parameterization in the xpcaarf tool, which will effectively reduce the derived fluxes by this factor; given the good agreement in the spectral index between PCA and the other measurements, this bootstrap approach represents the best available calibration of the total area.
} 


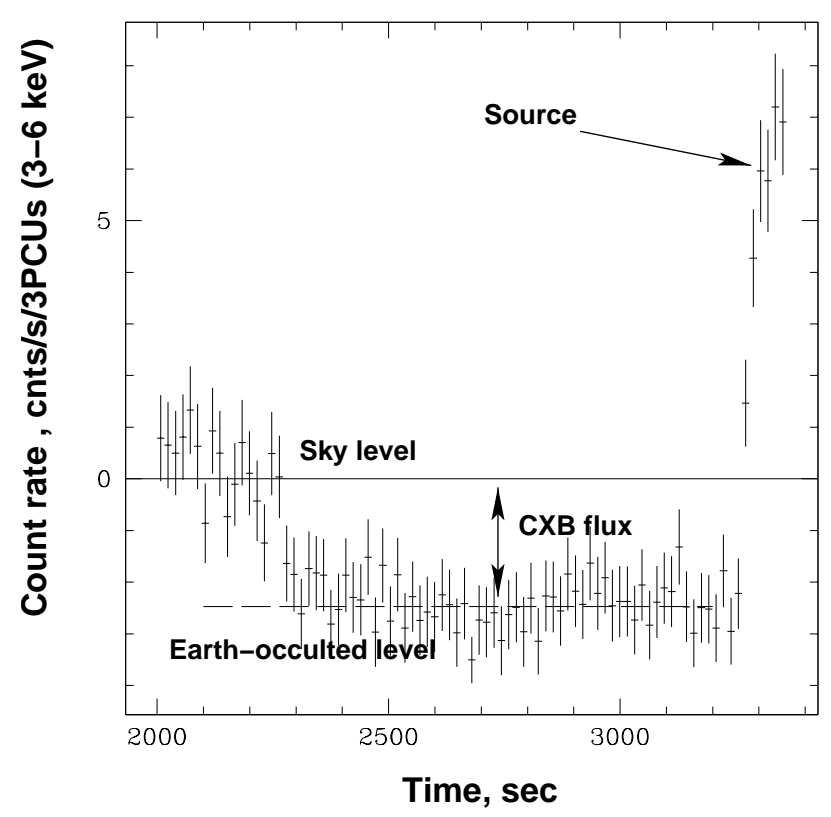

Fig. 1. Typical PCA background subtracted lightcurve during slew. The clear difference between the "sky" level and "Earth" level measures the CXB flux. The increase in countrate at the end of the lightcurve is caused by an X-ray source becoming un-occulted.

sky points; the net background rate is slightly different for the six points due to spatial fluctuations of the CXB.

Combination of Earth-occulted observations with sky observations provides the spectrum of the CXB. The Earth is treated as a shutter in front of the PCA. Figure 1 illustrates this point. The "sky" rate is approximately zero; the decrement between sky and occulted data is just the CXB flux.

\subsection{Map of the sky}

In order to obtain the spectrum of the CXB, we need to avoid the contamination from bright galactic and extragalactic sources present in the data. For this purpose we have constructed the map of the sky using the same Standard 2 data mode from which we collect the CXB spectrum.

The Standard 2 data mode of the PCA is present in all observations, maintains the maximum useful energy resolution, separates data by anode, and provides $16 \mathrm{~s}$ time resolution. The background-subtracted flux, measured by each PCU in the 3-20 keV energy band during each $16 \mathrm{~s}$ time bin was ascribed to the point of the sky where the optical axis of the RXTE/PCA was pointed at the middle of the time bin. Angular resolution is limited by the size of the PCA beam $\left(\sim 1^{\circ} \mathrm{FWHM}\right)$ and the movement of the optical axis during each $16 \mathrm{~s}$ interval. The typical velocity of the RXTE optical axis on the sky $\sim 0.1 \mathrm{deg} / \mathrm{s}-$ limits the spatial resolution along the slew direction $\sim 1-1.5^{\circ}$. The RXTE/PCA collimator field of view is $\sim 1^{\circ}$ (FWHM). Therefore the Standard 2 data provides a skymap with $\sim 1-1.5^{\circ}$ resolution. The map is presented in Fig. 2 .

Dark $\sim 1^{\circ}$ circles represent point sources, and the dark bar along the Galactic plane is caused by the Galactic ridge diffuse emission. Sco X-1 is not seen on the picture, because its strong
X-ray flux leads to violation of our criteria of filtering the "bad" data.

The $3 \sigma$ sensitivity of the obtained map to the point sources is approximately at the level of $\sim 10^{-11} \mathrm{erg} / \mathrm{s} / \mathrm{cm}^{2}$. At the present time this all-sky map is most sensitive in the energy band 3-20 keV. In addition to this, data of RXTE observations in the period 1999-2002 can provide us with approximately 2 times more effective exposure of the sky resulting in the all-sky map limiting sensitivity $\sim 0.5 \times 10^{-11} \mathrm{erg} / \mathrm{s} / \mathrm{cm}^{2}$. Analysis of the point sources is beyond the scope of this paper. We plan to present such analysis as a separate work.

Data within $1.5^{\circ}$ of all detected sources was masked. We have excluded the data obtained at low Galactic latitudes $(|b|<$ $20^{\circ}$ ) in order to avoid the influence of the Galactic ridge diffuse emission and weak Galactic X-ray sources. Regions of $10^{\circ}$ around LMC and SMC were also excluded.

Analysis of the latitude profiles of the Galactic ridge emission (Iwan et al. 1982; Valinia \& Marshall 1998; Revnivtsev et al. 2003) shows that its contribution to the detected X-ray flux at latitudes $|b|>20^{\circ}$ is less than approximately $10^{-2} \mathrm{cnts} / \mathrm{s} / \mathrm{PCU}$, and therefore negligible for our study (CXB has $\sim 2 \mathrm{cnts} / \mathrm{s} / \mathrm{PCU})$.

After exclusion of all mentioned regions we have approximately $1.7 \mathrm{Msec}$ of data, covering $\sim 55 \%$ of the sky $(\sim 22.6 \times$ $10^{3} \mathrm{deg}^{2}$ ) with non-zero exposure. Our sensitivity at higher energies is limited by the statistics of the background removal; our dark-earth spectrum contains only $25 \mathrm{ksec}$ of data.

\subsection{Spectrum of $C X B$}

The unfolded spectrum of CXB is presented in Fig. 3. The spectrum is well approximated by a single power law with a photon index $\Gamma=1.42 \pm 0.02$ and normalization $N=$ $9.8 \pm 0.3 \mathrm{phot} / \mathrm{s} / \mathrm{cm}^{2} / \mathrm{keV} / \mathrm{sr}$. If the slope is fixed at $\Gamma=1.4$, the normalization is $N=9.5 \pm 0.3 \mathrm{phot} / \mathrm{s} / \mathrm{cm}^{2} / \mathrm{keV} / \mathrm{sr}$. The observed flux of CXB in 3-10 keV energy band is $F_{3-10 \mathrm{keV}}=$ $(5.17 \pm 0.05) \times 10^{-8} \mathrm{erg} / \mathrm{s} / \mathrm{cm}^{2} / \mathrm{sr}$. The normalizations and flux are reported after the downward correction of 1.11 described above.

The study of CXB emission with PCA is limited by the accuracy of the instrumental background subtraction above $10 \mathrm{keV}$. Unmodelled variation in the PCA background averages $0.027 \mathrm{ct} / \mathrm{s} / \mathrm{PCU}$ in the $2-10 \mathrm{keV}$ band and $0.013 \mathrm{ct} / \mathrm{s} / \mathrm{PCU}$ in the 10-20 keV band (these figures are layer 1 only, Markwardt et al. 2002, and thus relevant to the data discussed here).

These errors were added in quadrature to the statistical errors of the measured count rates. The net statistical and systematic uncertainties of the measured CXB spectrum are shown by shaded area in Fig. 3.

\subsection{Cosmic variance}

Measurements of the CXB are also affected by spatial fluctuations, or cosmic variance, over the sky (see e.g. Barcons et al. 2000). Our CXB spectrum, which averages over a huge solid angle, is expected to represent a well defined average, although the background model, which is based on observations of only 


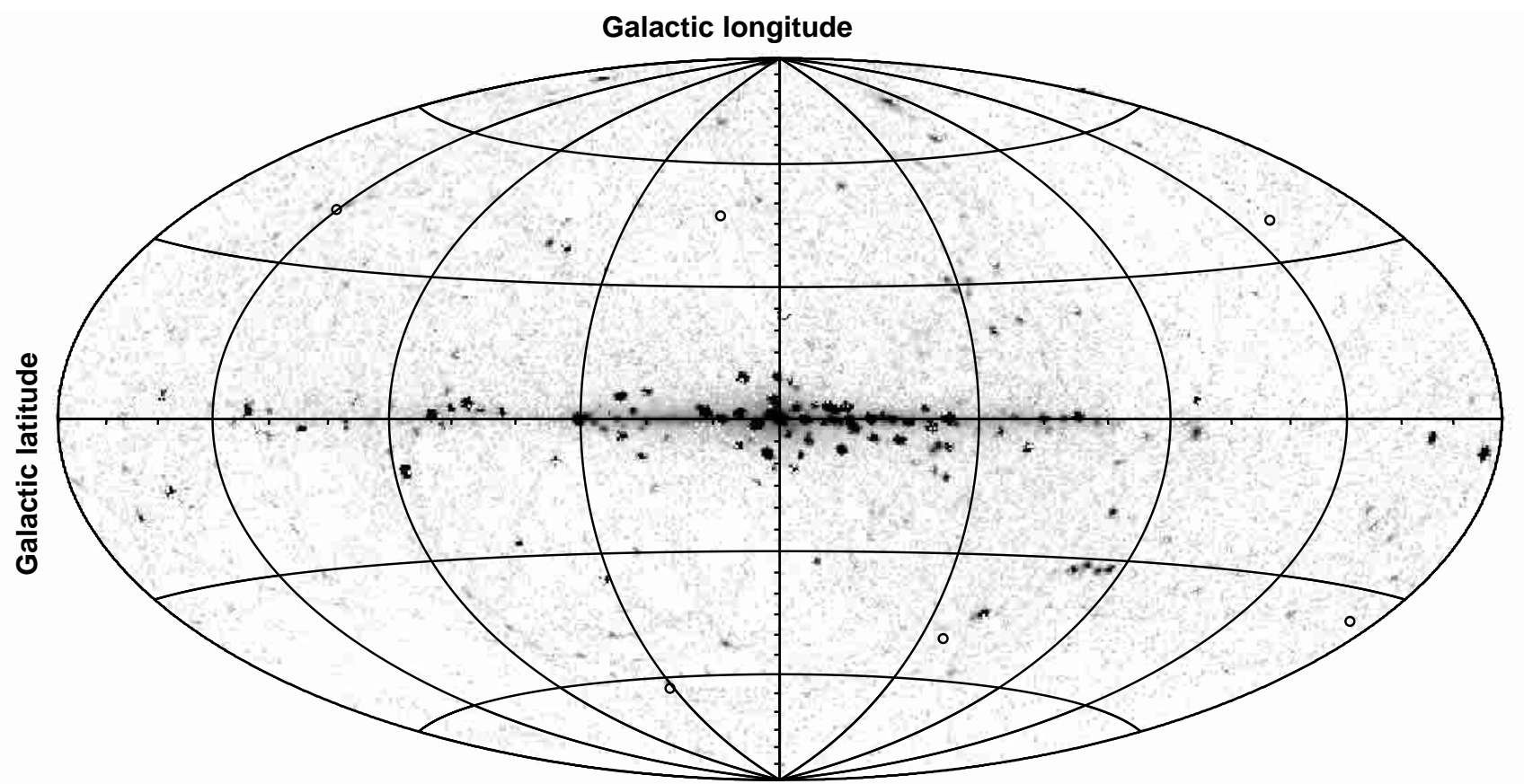

Fig. 2. Map of the sky, reconstructed from slew observations of RXTE/PCA. For this map we used data from layer 1 of PCUs $0,1,2,3-20 \mathrm{keV}$ energy band. The grid is separated by $45^{\circ}$ in $l$ and $30^{\circ}$ in $b$. Open circles indicate the regions used to construct the PCA background model.

6 distinct deep exposure points, may be affected. Other instruments that measure the CXB spectrum over smaller solid angles may be directly affected by this variance.

The data used to create the background model also allows a limited measurement of the fluctuations on the scale of the PCA beam of $\sim 1^{\circ}$. We analyzed data from the 6 "background" points (marked with open circles in Fig. 2). After subtraction of the PCA instrument plus average sky background, the spectra of these sky areas are significantly different. The individual spectra can be described with a common power-law index but differing normalization. The fractional root-mean-square amplitude of this variation in normalization is $7 \pm 1 \%$. The dashed lines in Fig. 3 show the $\pm 1 \sigma$ range of normalizations that would be measured over 1 square degree solid angles given this variation.

Field-to-field variation of the CXB is due to a combination of Poisson noise associated with different populations of sources drawn from the $\log N-\log S$ distribution and to large-scale structure. For the 1 degree field of view of the PCA, the Poisson variance is expected to be $\sim 4 \%$ (Barcons \& Fabian 1998; Barcons et al. 1998).

This suggests that the PCA background fields have measured large scale structure in the CXB, an interpretation consistent with Chandra measurements of the CXB fluctuations of $\sim 25-30 \%$ on scales of $\sim 0.07 \mathrm{sq} \mathrm{deg}$ (Yang et al. 2003), after scaling by the square root of the solid angle.

\section{Conclusion}

The large amount of slew data from the RXTE/PCA instrument allows us to study the spectrum and intensity of cosmic X-ray background averaged over a large solid angle.

After excluding areas around bright sources, the Galactic plane region $\left(|b|<20^{\circ}\right)$ and the regions of the Large

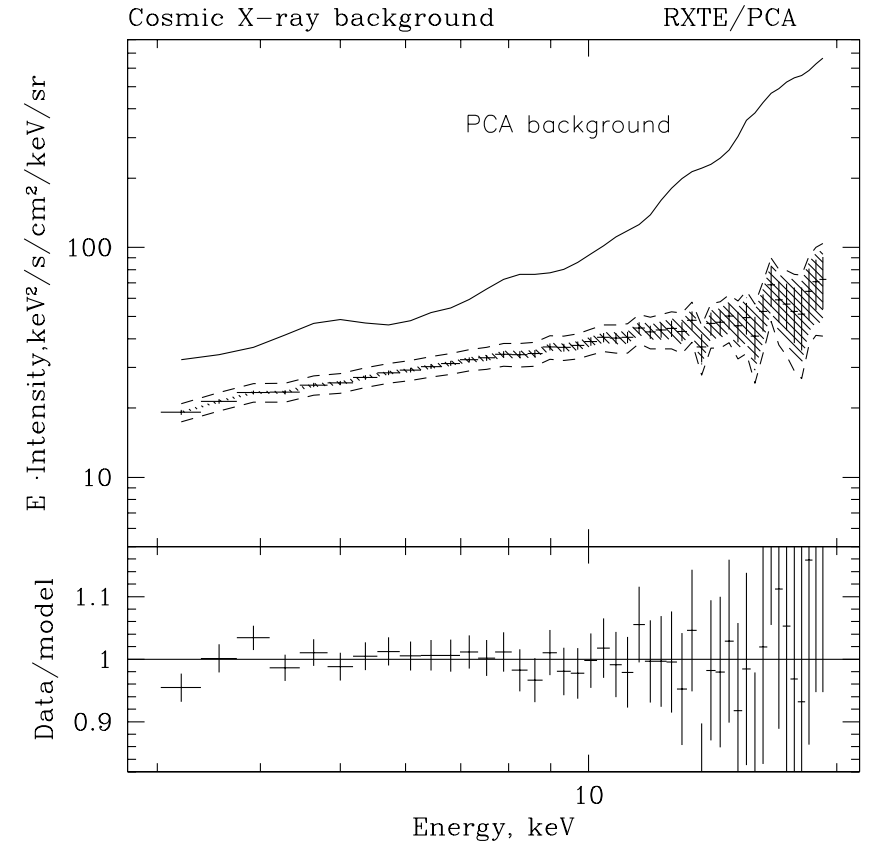

Fig. 3. Unfolded flux spectrum of CXB obtained by RXTE/PCA. Shaded area around the spectrum represents the amplitude of systematic uncertainties in the background subtraction. The spectrum of the PCA background (internal $+\mathrm{CXB}$ ) is shown by the solid line. Dashed lines represents root-mean-square amplitude of variations of normalization of CXB (cosmic variance) measured over different sky areas with effective solid angle $\sim 1 \mathrm{sq} \mathrm{deg}$. (see text). Lower panel shows the ratio of observed data to used model.

and Small Magellanic Clouds our data covers approximately $22.6 \times 10^{3} \mathrm{deg}^{2}$ of the sky. This data set measures, by definition, the average properties of the CXB. 


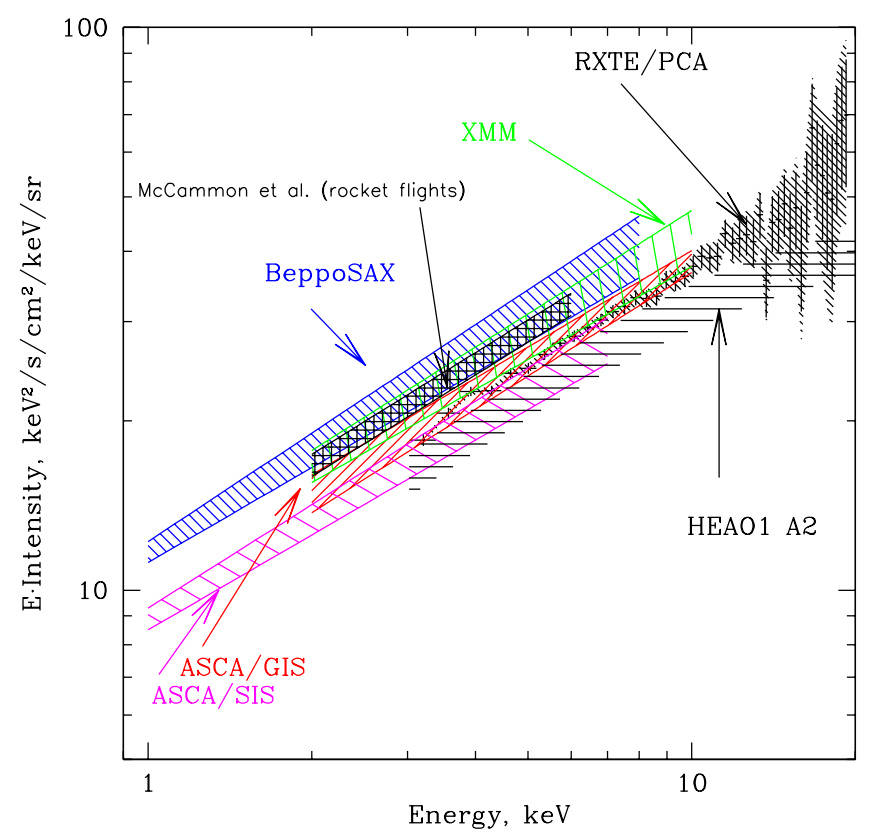

Fig. 4. Spectrum of CXB obtained by different instruments.

The spectrum of the CXB in the 3-20 keV energy band obtained from RXTE/PCA slew data is well approximated by a power law in the form $\mathrm{d} N(E) / \mathrm{d} E=N E^{-\Gamma}$ with photon index $\Gamma=1.42 \pm 0.02$ and normalization $N=9.8 \pm$ $0.3 \mathrm{phot} / \mathrm{s} / \mathrm{cm}^{2} / \mathrm{keV} / \mathrm{sr}$. Relatively large systematic uncertainties of the PCA instrumental background at $E>15-20 \mathrm{keV}$, and the decreasing ratio of CXB to instrument background did not allow us to study the spectrum above $\sim 20 \mathrm{keV}$, and the high energy cut-off detected by HEAO- 1 A2.

The slope of the CXB spectrum obtained by RXTE/PCA agrees well with that obtained by other observatories. The normalization value is slightly higher than, but marginally compatible with, HEAO-1 A2 (Marshall et al. 1980), obtained over a similarly large solid angle of the sky. The measurements of CXB normalization by different X-ray instruments give different variable values (Figs. 4 and 5), however, the weighted average of the imaging measurements gives a value that is inconsistent with HEAO-1 A2 (Barcons et al. 2000) and consistent with ours.

Our measurement relies on a scaling of the absolute area of the PCA to match the canonical value of the flux from the Crab nebula; as many experiments have used the Crab as a standard candle and as our measurement of the spectral shape is in good agreement, this should add no more than a few per cent uncertainty. Our result is marginally compatible both with the result from the xenon-filled, collimated proportional counters of HEAO-1 A2 and with the results from imaging instruments. However, observed subtle discrepancies between these measurements suggest that there are remaining systematic issues in the calibration of the effective area and/or solid angle in either the collimated or imaging experiments.

Acknowledgements. The authors are grateful to R. Mushotzky for valuable comments and suggestions. This research has made use of data obtained through the High Energy Astrophysics Science Archive

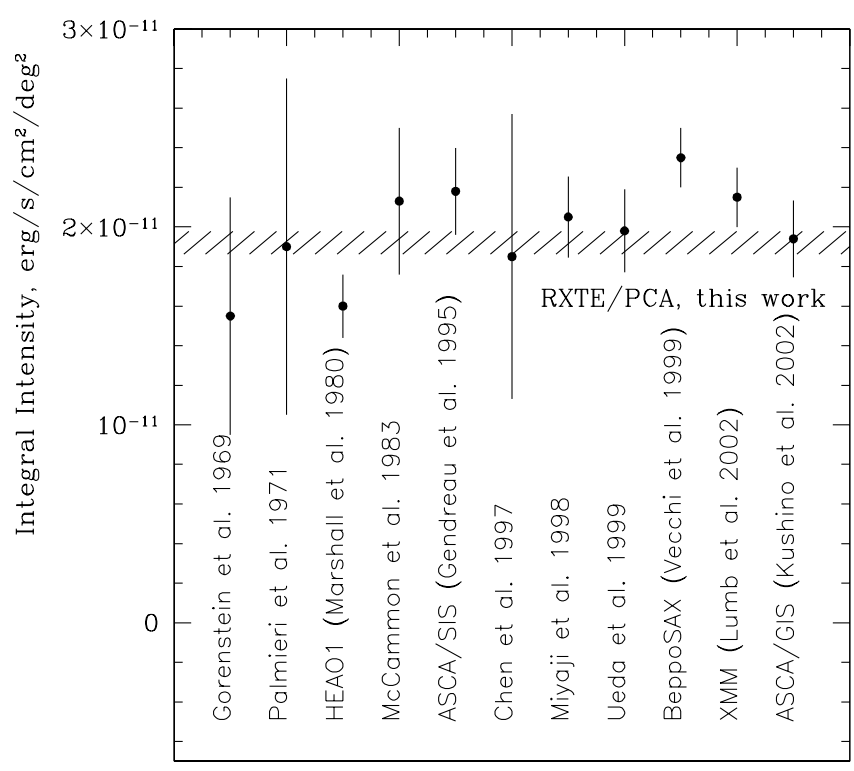

Fig. 5. Comparison of level of CXB obtained with RXTE/PCA with previous measurements.

Research Center Online Service, provided by the NASA/Goddard Space Flight Center.

\section{References}

Barcons, X., \& Fabian, A. C. 1998, Astr. Nachr., 319, 141

Barcons, X., Fabian, A. C., \& Carrera, F. J. 1998, MNRAS, 293, 60

Barcons, X. 1992, ApJ, 396, 460

Barcons, X., Mateos, S., \& Ceballos, M. T. 2000, MNRAS, 316, L13

Boldt, E. 1987, Phys. Rep., 146, 215

Bradt, H., Rotshild, R., \& Swank, J. 1993, A\&AS, 97, 355

Brandt, W., Alexander, D., Bauer, F., et al. 2003, HEAD meeting, 35

Chen, L.-W., Fabian, A., \& Gendreau, K. 1996, MNRAS, 285, 449

Fabian, A., \& Barcons, X. 1992, ARA\&A, 30, 429

Gendreau, K. C., Mushotzky, R., Fabian, A. C., et al. 1995, PASJ, 47, L5

Giacconi, R., \& Zamorani, G. 1987, ApJ, 313, 20

Giacconi, R., Zirm, A., Wang, J. X., et al. 2002, ApJS, 139, 369

Gorenstein, P., Kellogg, E. M., \& Gursky, H. 1969, ApJ, 156, 315

Gruber, D. 1992, in the X-ray background, ed. X. Barcons, \& A. Fabian (Cambridge: Cambridge Univ. Press), 44

Hasinger, G., Trümper, J., \& Schmidt, M. 1991, A\&A, 246, L2

Iwan, D., Shafer, R. A., Marshall, F. E., et al. 1982, ApJ, 260, 111

Jahoda, K., Swank, J. H., Giles, A. B., et al. 1996, Proc SPIE, 2808, 59

Kuulkers, E., den Hartog, P. R., in't'Zand, J. J. M., et al. 2003, A\&A, 399, 663

Kushino, A., Ishisaki, Y., Morita, U., et al. 2002, PASJ, 54, 327

Lumb, D. H., Warwick, R. S., Page, M., \& De Luca, A. 2002, A\&A, 389,93

McCammon, D., Burrows, D., Sanders, W., \& Kraushaar, W. 1983, ApJ, 269, 107

Markwardt, C., Jahoda, K., \& Smith, D. A. 2002, http://lheawww.gsfc.nasa.gov/users/craigm/pca-bkg/ bkg-users.html

Markwardt, C., Swank, J., Marshall, F., \& in't Zand, J. 2000, in ROSSI2000: Astrophysics with the Rossi X-ray Timing Explorer. March 22-24, 2000 at NASA's GSFC, Greenbelt, MD USA, E7 
Marshall, F., Boldt, E., Holt, S., et al. 1980, ApJ, 235, 4

Miyaji, T., Ishisaki, Y., Ogasaka, Y., et al. 1998, A\&A, 334, L13

Moretty, A., Campana, S., Lazzati, D., \& Tagliaferri, G. 2003, ApJ, 588,696

Mushotzky, R., Cowie, L. L., Barger, A. J., \& Arnaud, K. A. 2000, Nature, 404, 459

Palmieri, T. M., Burginyon, G. A., Grader, et al. 1971, ApJ, 169, 33

Pendleton, G., Paciesas, W., Fishman, G., et al. 1992, The Compton Observatory Science Workshop, GSFC, NASA, 47

Rees, M. 1980, Objects of High Redshift, IAU Symp., 92, 207

Revnivtsev, M., \& Sunyaev, R. 2002, Astr. Lett., 28, 69
Revnivtsev 2003, A\&A, 410, 865

Setti, G., \& Woltjer, L. 1989, A\&A, 224, L21

Seward, F. 1978, J. British Interplanet. Soc., 31, 83

Ueda, Y., Takahashi, T., Inoue, H., et al. 1999, ApJ, 518, 656

Valinia, A., \& Marshall, F. 1998, ApJ, 505, 134

Vecchi, A., Molendi, S., Guainazzi, M., et al. 1999, A\&A, 349, L73

Wu, X., Hamilton, T., Helfand, D. J., \& Wang, Q. 1991, ApJ, 379, 564

Yang, Y., Mushotzky, R., Barger, A., et al. 2003, ApJ, 585, L85

Zombeck, M. V. 1990, Handbook of Astronomy and Astrophysics, Second Edition (Cambridge, UK: Cambridge University Press) 